\title{
A Metrics Suite for Evaluating Flexibility and Complexity in Service Oriented Architectures
}

\author{
Mamoun Hirzalla ${ }^{1}$, Jane Cleland-Huang ${ }^{2}$, and Ali Arsanjani ${ }^{3}$ \\ ${ }^{1}$ DePaul University and IBM \\ mhirzallecs.depaul .edu \\ ${ }^{2}$ DePaul University \\ jhuang@cs. depaul.edu \\ ${ }^{3} \mathrm{IBM}$ \\ arsanjan@us.ibm.com
}

\begin{abstract}
Service Oriented Architecture (SOA) is emerging to be the predominant architectural style of choice for many organizations due to the promised agility, flexibility and resilience benefits. However, there are currently few SOA metrics designed to evaluate complexity, effort estimates and health status of SOA solutions. This paper therefore proposes a SOA metrics framework which includes both service level and SOA-wide metrics to measure design and runtime qualities of a SOA solution. The SOA-wide metrics predict the overall complexity, agility and health status of SOA solutions, while service level metrics focus on the fundamental building blocks of SOA, i.e. services. The combined views deliver a compelling suite of SOA metrics that would benefit organizations as they consider adopting SOA. These metrics, which are based on observations of many SOA engagements, are illustrated through a case study that describes a recent ongoing project at IBM where SOA was utilized to build the solution assets.
\end{abstract}

Keywords: SOA metrics, SOA complexity, agility, flexibility, SOA health.

\section{Introduction}

Service Oriented Architecture (SOA) is becoming an increasingly popular architectural style that focuses on providing the right tools and methods for building distributed applications. The fundamental building blocks of SOA are repeatable business tasks realized as services and implemented in a variety of distributed components such as CORBA, EJBs and web services [9].

From a business perspective, one of the primary objectives of SOA-based systems is the alignment between business and IT and the flexibility and business agility that SOA injects into an organization [9]. This is achieved through systematically designing and building a SOA-based solution using a method such as SOMA, invented and initially developed in IBM [7]. SOMA defines key techniques and provides prescriptive tasks and detailed normative guidance for analysis, design, implementation, testing, and deployment of services, components, flows, information, and policies needed 
to successfully design and build a robust and reusable SOA solution in an enterprise [7]. However SOMA, like other SOA-based methodologies, does not provide techniques or metrics for measuring underlying complexity and flexibility qualities of a SOA-solution.

This paper therefore proposes a new suite of metrics, designed specifically to evaluate flexibility and agility versus complexity of a SOA solution. The set of metrics can be used to provide a diagnosis for the health of a SOA solution by providing information about services used within a SOA solution, their composition interfaces or provided operations, architectural decisions, flexibility, agility and complexity. The term "health" of SOA solutions is not limited to the metrics or characteristics identified in this paper. Obviously, there are other elements that must be considered to determine the overall health of a SOA solution. SOA Governance with its emphasis on security, management and testing is a major factor to consider when evaluating such a question. This paper will focus on the architectural considerations as they pertain to the "health" factor.

The proposed SOA metrics are grouped into two major categories: design-time SOA metrics and run-time SOA metrics. Table 1 summarizes the metrics categories and their applicability.

Table 1. Individual Design-time and Run-time SOA Metrics

\begin{tabular}{|l|l|l|}
\hline Classification & Metric Name & Metric Applicability \\
\hline Design-time & Weighted Service Interface Count (WSIC) & Service \\
\hline Design-time & Stateless Services (SS) & Service \\
\hline Design-time & Service Support for Transactions (SST) & Service \\
\hline Design-time & Number of Human Tasks (NHT) & Service \\
\hline Run-time & Number of Services (NOS) & SOA Solution \\
\hline Run-time & Service Composition Pattern (SCP) & SOA Solution \\
\hline Run-time & Service Access Method (SAM) & SOA Solution \\
\hline Run-time & Dynamic vs. Static Service Selection (DSSS) & SOA Solution \\
\hline Run-time & Service Realization Pattern (SRP) & Service \\
\hline Run-time & Number of Versions Per Service (NOVS) & Service \\
\hline
\end{tabular}

These metrics were identified as a result of experiences gained from engaging in building numerous SOA solutions. More traditional object-oriented (OO) metrics can also be used to evaluate the internal complexities of individual services [6]. Although, each metric is individually reported, results are also aggregated in terms of three indices, a general SOA Complexity Index (SCI), a Services Complexity Index (SVCI), and a Flexibility and Agility Index (FAI). Table 2 provides a list of the proposed SOA indices and their descriptions.

These metrics can be captured as part of the SOMA lifecycle. Design-time metrics are gathered during the identification, specification, and realization phases, while runtime metrics are gathered during implementation and deployment. Furthermore, metrics collection is not difficult, because metrics can be automatically collected through inspecting SOA artifacts such as a service's Web Service Description Language (WSDL) file, Choreography Description File (CDL), related policies documents, or through inspecting SOAP messages and source code.

The remainder of this paper is laid out as follows. Section 2 provides a survey of SOA metrics, and explains why OO metrics are useful yet insufficient for measuring 
SOA solutions. Section 3 introduces and describes SOA design-time metrics. Section 4 introduces and describes SOA runtime metrics. Section 5 demonstrates the usefulness of the metrics through a case study taken from a recent ongoing project that utilized SOA to build the solution assets. While this case study does not empirically validate the proposed metrics, it illustrates how they could be reasonably used to evaluate the complexity and agility of a SOA solution and provide some indications regarding the health of a SOA solution. Finally, section 6 concludes with a discussion for future work.

Table 2. Aggregate SOA Indices

\begin{tabular}{|l|l|}
\hline SOA Index & Description \\
\hline $\begin{array}{l}\text { SOA } \\
\text { Complexity } \\
\text { Index (SCI) }\end{array}$ & $\begin{array}{l}\text { Measures the inherent complexity of the SOA solution including its security, } \\
\text { management and SOA governance measures, all of which offer significant benefits but } \\
\text { also increase the complexity of the overall SOA }\end{array}$ \\
\hline $\begin{array}{l}\text { Services } \\
\text { Complexity } \\
\text { Index } \\
\text { (SVCI) }\end{array}$ & $\begin{array}{l}\text { Measures complexity, but looks at the individual complexities of each of the composed } \\
\text { services }\end{array}$ \\
\hline $\begin{array}{l}\text { Flexibility } \\
\text { and Agility } \\
\text { Index (FAI) }\end{array}$ & $\begin{array}{l}\text { Tracks the flexibility and agility of the SOA solution, which represent SOA's primary } \\
\text { objective to bring business agility and flexibility to an organization [4] }\end{array}$ \\
\hline
\end{tabular}

\section{Background Information}

Measurement is an important component of any process improvement initiative. Software metrics enable qualities of interest to be measured and evaluated, in order to identify potential problems, and to provide insight into the costs and benefits of a potential solution. Unfortunately current SOA metrics are relatively immature and tend to suffer from many of the problems previously identified by Chidamber and Kemerer in respect to early OO metrics. These problems include lack of desirable measurable properties, over generalization, focus on specific technologies, and collection difficulty [6].

Although OO metrics [6] can be used to measure the internal complexity of a service built on the OO paradigm; they are not sufficient for measuring more global SOA qualities. A few researchers have proposed various SOA metrics. For example, Liu et al. [2] developed complexity and attackability metrics and showed that complexity has a negative impact on security. Their Average Service Depth metric computes the average number of dependency relationships per atomic service node, as representatives of various software capabilities within a system. Rud et al. [1] focused on the infrastructure and performance aspects of SOA solutions and identified many SOA metrics that are granular in nature. These metrics were classified into the three major areas of complexity, criticality and reliability, and performance metrics. They identified a relationship between complexity of a service and amount of time required to build such a service. Qian et al. [3] developed decoupling metrics for SOA software composition such as Average Service State Decomposition (ASSD), Average Service Persistent Dependency (ASPD) and Average Required Service Dependency (ARSD), and used it to evaluate decoupling between service-oriented components in 
the service composition such as Business Process Execution Language (BPEL); a useful set of metrics that should be considered for loose coupling considerations as part of the health status of SOA solutions.

Unfortunately, none of these metrics provide a comprehensive approach for measuring flexibility and agility which represent significant factors in the short and longterm success of a SOA solution. In contrast, the metrics proposed in this paper are specifically designed to evaluate the impact of SOA architectural decisions upon the flexibility, agility and complexity of a SOA solution.

\section{SOA Design-Time Metrics}

The four metrics defined in this section measure the flexibility, agility, and complexity of the solution in respect to design time decisions. The metrics are independent of the underlying code, and could be applied to either JEE web services or .Net services. Metrics are first computed for individual services and then compiled into a more global metric and applied to the FAI, SVCI, and SCI indices.

\section{Metric 1: Weighted Service Interface Count (WSIC)}

Definition: WSIC $=$ The weighted number of exposed interfaces or operations per service as defined in the WSDL documents. The default weight is set to 1 . Alternate weighting methods, which need to be validated empirically, can take into consideration the number and the complexity of data types of parameters in each interface. In the default case, WSIC simply returns a count of the number of exposed interfaces or methods defined in the WSDL documents.

Hypothesis: The higher the number of service interfaces the more complex a service becomes and by association the more complex a SOA solution becomes. In addition, there is a direct relationship between the complexity of the exposed interfaces and the complexity of the data structures required per interface.

Observations: The greater the number of defined interfaces per service within a SOA solution the more complex a service becomes due to the following factors. (i) The amount of work required to specify, construct and test every interface on the service increases. (ii) The amount of monitoring required to ensure that service level agreements (SLAs) are met increases with every invocation of an interface. (iii) With the increase in complexity of individual interfaces of the data structures for a given service, performance and problem determination concerns may become a primary issue. Performance and root cause issues are hard to predict and diagnose.

Impact on defined indices: Both SVCI and SCI increase as WSIC increases. There is no impact on the FAI index.

\section{Metric 2: Stateless Services (SS)}

Definition: $\mathrm{SS}=$ The fraction of services which are stateless (SLS) as opposed to stateful (SFS) as defined in the Web Services Resource Framework (WS-RF) [8] or WS-Context [12]. SS $=$ SLS $/($ SLS + SFS $)$. 
Hypothesis: Developing stateful web services is much harder than developing stateless web services and therefore increases the complexity of a given service.

Observations: Both WS-RF and WS-Context define how to support stateful interactions while using the web services programming model. WS-RF follows a resourcebased approach to support state, while WS-Context uses a context that resembles shared state management across different interacting web services. Regardless of which approach is used, supporting transactions in web services will add an additional layer of complexity to programming web services. Complexity also increases with an increase in the number of web services that are participating in a stateful interaction.

Impact on defined indices: SVCI increases as the raw count of SFS services increase. Both SCI and FAI increase with decreased SLS values, i.e. when the fraction of stateless services increases. SCI would increase by a higher value if SLS fraction of stateless services decrease compared to stateful services.

\section{Metric 3: Service Support for Transactions (SST)}

Definition: SST = The fraction of transaction-aware services (TAS) in relation to the overall number of transaction-aware and non-transaction aware (NTAS) services within the SOA solution. SST $=$ NTAS/(NTAS+TAS)

Hypothesis: Web services supporting transactions are more complex to build and as a result increase the overall complexity of SOA solutions.

Observations: Traditional transaction systems use a two-phase commit protocol to achieve atomicity between transaction participants. Support of transactions in web services is accomplished through support of the WS-TX specification which includes the WS-Coordination, WS-Atomic Transaction and WS-Business Activity specifications [10]. The WS-TX specification requires additional code that needs to be included in the body of a web service and its invoking client. In order to maintain consistency of transactions, compensating transactions are required to provide correct compensation actions. Furthermore, the coordination between transaction-aware services requires additional effort that injects additional complexity into building transaction-aware services and SOA solutions.

Impact on defined indices: SVCI increases as the raw count of transaction-aware services increase. SCI increases as SST values increase, i.e. when the fraction of transaction-aware services increases. FAI is relatively unaffected by support for transactions in web services. However, extensive use of transactions is likely to constrict how flexible and agile a SOA solution becomes.

\section{Metric 4: Service Realization Pattern (SRP)}

Definition: SRP $=$ The fraction of services that are realized through Indirect Exposure (IE) in respect to the total number of services that are realized using both IE and Direct Exposure (DE). SRM = IE /(IE+DE)

Hypothesis: The more indirect exposure service realizations in SOA solution, the more complex a service becomes and by association the more complex a SOA solution becomes. 
Observations: There are many service realization patterns that can be used for exposing and using services including the two primary patterns of Direct Exposure (DE) and Indirect Exposure (IE). DE refers to exposing current IT systems or modules as a service without having to go through an intermediary component. For example, a stored SQL procedure could be turned into an information service directly by wrapping it through a web service and exposing the web service to consuming clients. Indirect Exposure, on the other hand, refers to exposing current IT systems or a module as a service by going through an intermediary component such as an EJB. Direct Exposure services provide a much faster method for creating and invoking services. They also require less time to decide on appropriate interfaces since they tend to match the interfaces that can be exposed from the legacy asset. Direct Exposure services also require less time to develop and test due to the direct connectivity with the backend system. In comparison, Indirect Exposure realization of services entails additional IT components to mediate between a service and an IT asset. While this provides additional flexibility to the overall SOA solution, it also increases the time to build and test such services, and requires additional management and monitoring steps to ensure services and their associated components are functioning properly.

Impact on defined indices: SVCI increases with the use of IE realization of services and decreases with the use of DE realization. SCI will increase with the increase in the value of the SRP. The higher the ratio of DE to IE realizations, the less complexity. This is inversely related to the ratio of IE services to the overall number of both DE and IE services. In other words, the lower the ratio, the less complexity. FAI depends on the ratio of DE services to the overall number of both DE and IE services. The lower the ratio, the more flexible and agile a SOA solution will be. This is inversely related to the ratio of IE services to the overall number of both DE and IE services. In other words, the lower the ratio, the less complexity.

\section{Metric 5: Number of Human Tasks (NHT)}

Definition: NHT $=$ The fraction of tasks as part of a business flow that are manual.

Human Tasks (HT) in a process flow are important due to their need in real life scenarios. For example, a business process flow can invoke many services to automate a banking process that requires the final verification of an auditor through a human interaction. The judicious use of human tasks within a process is accepted as a fact of life. However, with the increased use of human tasks, we end up with less flexible processes within a SOA solution. NHT is computed as NHT $=\mathrm{HT} /(\mathrm{HT}+\mathrm{AT})$ where AT refers to an automated task.

Hypothesis: The use of too many Human Tasks in a SOA solution increases complexity and decreases flexibility of a SOA solution.

Observations: BPEL defines business processes as collections of activities that invoke services. BPEL doesn't distinguish between services that are provided by applications and other interactions, such as human interactions. From our metrics perspective, any human task that is being executed as part of a BPEL flow within a SOA solution will have an impact on both complexity and flexibility. 
Impact on defined indices: There is no direct impact on the service complexity due to the use of human tasks. However, the maintenance issues associated with manual tasks will increase the overall complexity of such tasks and therefore increasing the overall value of the SCI. In addition, too many human tasks will negatively impact the flexibility and agility of the overall SOA solution and decreases the value of the FAI.

\section{SOA Run-Time Metrics}

SOA run-time metrics measure the overall complexity and agility of a SOA solution. As a result, SOA metrics help in achieving the overall objective of exposing overly complex SOA architectures and provide insights into the flexibility factor of a SOA solution. In order to calculate the SOA run-time metrics, multiple SOA components are considered such as ESB, registry, and SOA governance.

\section{Metric 6: Number of Services (NOS)}

Definition: $\mathrm{NoS}=$ Total number of services that comprise a SOA solution

Observations: Greater numbers of services increase the complexity of a SOA solution due to the following factors. (i) An increase in the amount of work required to identify, specify, create, test, deploy and maintain such services. (ii) The need to provide better SOA Governance and service lifecycle management for the services within a SOA solution becomes more critical as the number of services used within a SOA solution increase. (iii) The increased number of services within a SOA solution usually places additional demand on the SOA infrastructure where services are deployed to meet service level agreements (SLAs) and scalability requirements.

Hypothesis: The higher number of services within a SOA solution, the more complex a SOA solution becomes.

Impact on defined indices: SVCI is not impacted. SCI increases as the number of services increase.

\section{Metric 7: Service Composition Pattern (SCP)}

Definition: $\mathrm{SCP}=$ The fraction of web services which are composite.

An Atomic Service (AS) is a web service that can stand on its own and does not require the use of other web services to complete its functionality. In contrast Composite Services (CS) are composed of other web services through aggregation, also referred to as structural composition [12] (CSs), or at runtime through invocation and orchestration of services through use of a workflow (CSwf). SCP is computed as SCP = CS / $(\mathrm{AS}+\mathrm{CSs}+\mathrm{CSwf})$ and can be further refined according to the composition method. SCPs measures the fraction of composite services constructed using aggregation, while SCPwf measures the fraction composed at runtime. These two metrics are defined as follows: $\mathrm{SCPs}=\mathrm{CSs} /(\mathrm{AS}+\mathrm{CSs})$ and SCPwf $=\mathrm{CSwf} /(\mathrm{AS}+\mathrm{Cswf})$.

Hypothesis: The use of composite services in a SOA solution increases both complexity and flexibility of a SOA solution.

Observations: Service orchestration refers to the collaboration among services that is driven by a central component or workflow engine, while service choreography refers 
to the collaboration among different parties that are each responsible for one or more steps of service interaction. The complexity of choreography stems from the fact that no central entity controls the process as a whole and therefore gleaning insight into the overall picture of the process status requires additional effort. Managing the state of service choreography often leads to complications due to the many events that need to be triggered and correlated to ensure proper execution of business functionality.

Impact on defined indices: There is no impact on the SVCI for an atomic service. However, structural composition will increase the value of the SVCI. An increase in SCP leads to an increase in both FAI and SCI.

\section{Metric 8: Service Access Method (SAM)}

Definition: SAM = The fraction of services accessed using a virtualization layer, referred to as Virtualized Access Services (VAS), in respect to the total number of services that are VAS or accessed point to point(PPS). SAM = VAS / ( VAS + PPS )

Hypothesis: Virtualized access to services within a SOA solution increases the flexibility and agility of a SOA solution but also increases its complexity.

Observations: Services can be accessed directly by an invoking client or through a broker component, referred to as an Enterprise Service Bus (ESB) which looks up the address of required services through a registry component, retrieves the Web Service Definition Language (WSDL) file, and then binds to that service during the invocation process. The ESB in essence provides a virtualization layer so that invoking clients do not need to know individual physical addresses of services. The ESB is responsible for routing and translating requests and responses among service requestors and service providers. The method of invocation will be referred to as Virtualized Access Services (VAS). The invocation of services also plays a role in the level of complexity associated with this metric. Services that are invoked directly are considered point to point (PPS) connections and are harder to maintain. On the other hand, services invoked through an ESB are easier to maintain but more complex to setup, because adding an ESB component to the overall SOA solution is not a simple task. It requires proper planning and design of the ESB and interacting services. The inclusion of a service registry is not considered a factor for this metric since it is dependent on the level of SOA governance and management required as part of the overall SOA solution, and is likely to be introduced when the number of services exceeds a threshold level.

\section{Impact on defined indices:}

There is no impact on SVCI. Over the long-term SCI decreases as SAM increases, i.e. use of point to point connections increase. FAI also decreases as SAM increases.

\section{Metric 9: Dynamic vs. Static Service Selection (DSSS)}

Definition: DSSS = The number of services that are selected dynamically (DS) over the total number of services that are selected dynamically or statically (SS). DSSE $=$ DS/(DS+SS).

Hypothesis: The more dynamic selection of services for execution within a SOA solution the more complex, flexible and agile a SOA solution becomes. 
Observations: Service consumers can invoke services through the typical stub and tie classes that are generated by the available tools in the market place. However, there are instances where the business logic requires a truly dynamic method for invoking the proper service based on a business rule that mandates a better service for a platinum-level customer to maintain their loyalty. In such scenarios, having a broker in the middle and the proper advertising of available services becomes mandatory. For this kind of scenario, the ESB and registry along with service monitoring software will play a significant role. However, it increases the overall complexity of maintaining a SOA solution. As Dynamic service invocation provides better flexibility and agility since a process can adapt much quicker than a process those hard codes static locations of services.

Impact on defined indices: There is no impact on SVCI. The higher the ratio of SSE, the higher the complexity of a SOA solution; SCI therefore increases as SSE increases. Moreover, the higher the ratio of SSE, the more flexible a SOA solution.

\section{Metric 10: Number of Versions per Service (NOVS)}

Definition: NOVS $=$ The total number of versions over the total number of services within the SOA solution. NOVS = VERSIONS / SERVICES

Hypothesis: The higher the number of service versions the more complex a service becomes and by association the more complex a SOA solution becomes.

Observations: The number of versions available in production per service is dependent on the level of change that services undergo while they are in production. It may signal an unstable service interface in the first place and a situation where services were rushed into production. The greater the number of available versions per service within a SOA solution the more complex a service becomes due to the following factors. (i)The amount of work required to keep track of service versions and their associated clients. Multiple service versions may also provide different SLAs for different consumers. The SOA Governance and management aspects of tracking service versions will become harder with every new service version that is maintained. (ii) The amount of regression testing required per service version increases if a common defect is discovered in one of the service versions. Therefore, additional time is required in order to ensure that all service versions are operating uniformly for similar business logic. The proliferation of many versions for the same service may point to lack of proper design that considers the level of reuse required. Reusable services tend to be more carefully planned, designed and implemented.

Impact on defined indices: Both SVCI and SCI increase as NVS increases. There is no impact on FAI.

\section{Case Study}

The metrics described in this paper still need to be empirically evaluated across multiple SOA applications to determine if they are complete and relatively non-redundant. In this section we briefly describe their use in a case study which is representative of multiple internal projects developed at IBM. The customer in the case study is a bank that is considering a SOA solution to address some of the primary pain points related to its Account Open business process. The current process was riddled with manual tasks 
that were causing the Account Open process to extend to 14 days instead of 10 minutes as offered by the bank's primary competitors.

One of the primary business objectives of the bank's SOA Solution was to inject agility and flexibility into the Account Open business process while trying to minimize complexity of the overall SOA solution. At a high level, the Account Open business process consisted of two primary sub-processes: Account Verification and Account Activation.

SOMA was used to model the optimized business process and identify and specify the services needed to realize the new business process vision. Candidate services were identified using SOMA's top down and bottom up approaches and SOMA's service litmus test (SLT) was applied to rationalize and verify candidate services. These services were developed from existing assets or else programmed from scratch. The SOA solution was built in three iterations. The first iteration of service creation focused on creating new services that were used to automate previous manual tasks such as credit check and address validation. The second service connectivity iteration focused on integrating disparate systems within the bank through the incorporation of an ESB to virtualize access to services and enhance the overall flexibility of the solution. Finally the Interaction and Collaboration iteration provided an enhanced user interface to the web channel by incorporating the automated steps of the newly optimized Account Open business process with the created services.

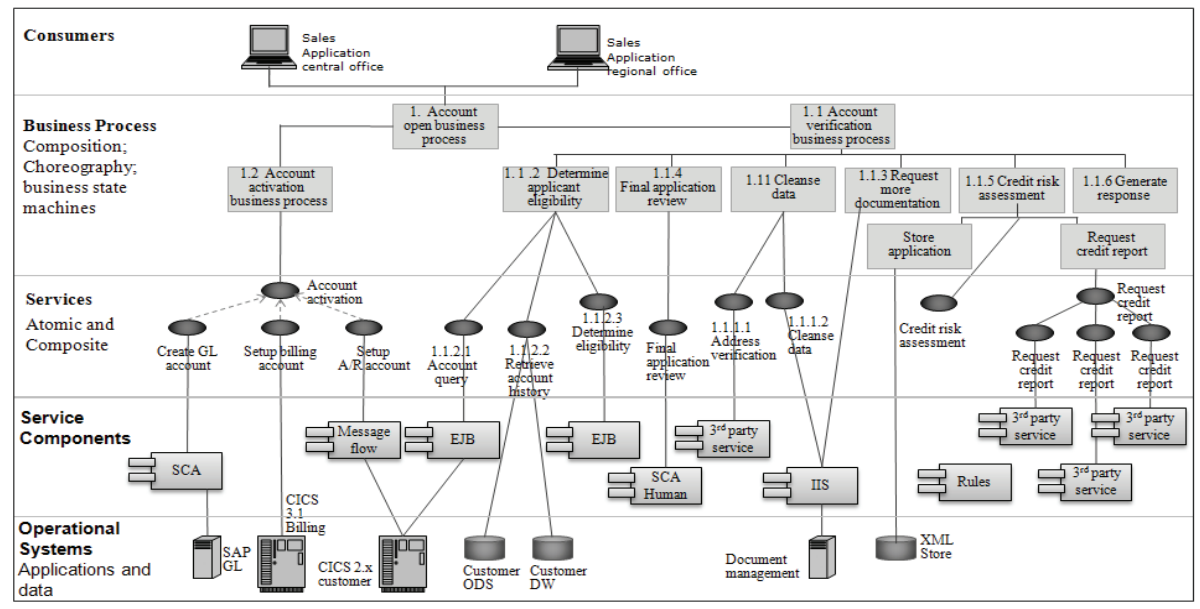

Fig. 1. Account Open SOA Solution Stack

Figure 1 provides a quick overview of the solution stack for the SOA solution. Identified services were utilized through the business process layer. Some services used the indirect exposure realization pattern while other services connected directly to backend systems. The solution stack does not reflect the physical architecture of the solution. The physical architecture of the solution included a layer for the ESB to virtualize access to services.

Table 3 provides an overview of the services used for the Account Open SOA solution. The Interaction and Collaboration (I\&C) iteration was the most complex due to the total number of services used within the iteration and the utilized service realization 
patterns. The (I\&C) iteration's SRP metric value is equal to 1 which is the highest value for the metric. This indicates higher complexity levels for service development since services are using an additional indirection layer to complete their capabilities. On the other hand, flexibility and agility index is higher for the same indirection reasons mentioned earlier due to the loose coupling that indirect exposure injects into a SOA solution.

Table 3. Services and metrics from the Open Account case study

\begin{tabular}{|c|c|c|c|c|c|c|c|c|}
\hline $\begin{array}{l}\text { Scenario } \\
\text { Service Creation }\end{array}$ & Service Name & WSIC & $\begin{array}{l}\text { NOS } \\
0.83\end{array}$ & 0.17 & $\begin{array}{l}\text { SCP } \\
0.17\end{array}$ & $\begin{array}{c}\text { DSSS } \\
0.17\end{array}$ & $\begin{array}{c}\text { SRP } \\
0.5\end{array}$ & $\begin{array}{c}\text { NHT } \\
0\end{array}$ \\
\hline & Billing Account Setup Service & 1 & Internal & Internal & Atomic & Static & DE & Automated \\
\hline & AR Account Inquiry Service & 1 & Internal & Internal & Atomic & Static & IE & Automated \\
\hline & AR Account Setup Service & 1 & Internal & Internal & Atomic & Static & IE & Automated \\
\hline & Determine Applicant Eligibility Service & 1 & Internal & Internal & CSwf & Static & $\mathrm{IE}$ & Automated \\
\hline & Credit Report Request Service & 1 & Internal & Internal & Atomic & Static & $\mathrm{DE}$ & Automated \\
\hline & Address Verification Service & 1 & External & External & Atomic & Dynamic & $\mathrm{DE}$ & Automated \\
\hline \multirow[t]{10}{*}{$\begin{array}{l}\text { Interaction and } \\
\text { Collaboration }\end{array}$} & & & 1 & 0 & 0 & 0 & 1 & 0.11 \\
\hline & Customer Data Cleanse & 2 & Internal & Internal & Atomic & Static & IE & Automated \\
\hline & Get Customer Selection of Account & 1 & Internal & Internal & Atomic & Static & $\mathrm{IE}$ & Automated \\
\hline & Account Rep Review Account Service & 1 & Internal & Internal & Atomic & Static & $\mathrm{IE}$ & Automated \\
\hline & Message Customer Waiting for Notification & 1 & Internal & Internal & Atomic & Static & $\mathrm{IE}$ & Automated \\
\hline & Request More Documentation Service & 1 & Internal & Internal & Atomic & Static & IE & Automated \\
\hline & Complete Documentation Request Service & 1 & Internal & Internal & Atomic & Static & $\mathrm{IE}$ & Automated \\
\hline & Final Application Review Service & 1 & N/A & $\mathrm{N} / \mathrm{A}$ & $\mathrm{N} / \mathrm{A}$ & N/A & N/A & Human \\
\hline & Notify Customer with Decline & 1 & Internal & Internal & Atomic & Static & $\mathrm{IE}$ & Automated \\
\hline & Notify Customer with Acceptance & 1 & Internal & Internal & Atomic & Static & $\mathrm{IE}$ & Automated \\
\hline \multirow[t]{6}{*}{ Service Connectivity } & & & 1 & 0 & 0.2 & 0 & 0.8 & 0 \\
\hline & Setup Billing Account Service & 1 & Internal & Internal & Atomic & Static & $\mathrm{DE}$ & Automated \\
\hline & Account Activation & 4 & Internal & Internal & CSs & Static & $\mathrm{IE}$ & Automated \\
\hline & Inquiry AR Customer Account Service & 1 & Internal & Internal & Atomic & Static & $\mathrm{IE}$ & Automated \\
\hline & Setup AR Account Service & 1 & Internal & Internal & Atomic & Static & $\mathrm{IE}$ & Automated \\
\hline & Create GL Account Service & 2 & Internal & Internal & Atomic & Static & $\mathrm{IE}$ & Automated \\
\hline
\end{tabular}

Notes:

- All services were stateless, provided no support for transactions, had only a single version, and utilized virtualized access. These columns are therefore not shown in the table.

- The NOS metric column contains two values since the metric distinguishes between the ratios of external services vs. internal services relative to the overall number of services.

The I\&C iteration produced less flexibility due to the existence of a manual human task, however this was seen as a necessary tradeoff because a human needed to verify the final steps of the account for a certain percentage of applicants. This is compensated for by the SRP metric since all I\&C iteration service realization patterns are done through indirect exposure which provides looser coupling for the overall SOA solution.

The determination of health of the SOA application based on the short analysis that was completed is a more complex question given the limited amount of data. As indicated earlier in this paper, additional SOA governance parameters need to be evaluated. However, from the limited information that we collected for this case study, there are no red flags that can be raised to indicate an unhealthy behavior or any major issues with the health of the given SOA solution.

\section{Future Work}

The primary contribution of this paper is the proposed set of metrics that should be tracked and measured for every SOA engagement so that better insight can be gleaned into the complexity, agility and flexibility of the SOA application. However, one of the common problems with new metrics suite is the difficulty of empirically validating and calibrating them across a number of projects. For example, the case study demonstrates 
the need to weight each metric to provide more accurate complexity, flexibility, and agility measurements. For example, table 3 shows a slightly higher value of SCP for the Service Connectivity iteration. It also shows a positive value for DSSS metrics which tends to increase complexity. By definition, this should have resulted in more complexity for the Service Connectivity which is true. However, from real life experience the Service Connectivity iteration took longer to accomplish due to increased complexity as a result of other metrics in the table such as SRP and WSIC. Despite these problems, this paper has proposed a reasonable set of metrics, identified as a result of observing numerous SOA deployments. Future work will include empirical assessment of these metrics and the identification of additional ones in order to build a demonstrably useful set of SOA metrics for predicting complexity, flexibility, and agility across a broad spectrum of SOA applications. Additional work is also required to provide clear methods for calculating the values of the proposed aggregate SOA indices and interpret their implications in terms of SOA flexibility, agility and complexity.

Acknowledgments. We would like to thank our colleague Russell Hartmann from IBM for his help in providing the data for the case study.

\section{References}

1. Rud, D., Schmietendorf, A., Dumke, R.R.: Product Metrics for Service-Oriented Infrastructure. In: International Workshop on Software Measurement/Metrikon 2006 (2006)

2. Liu, Y.M., Traore, I.: Complexity Measures for Secure Service-Oriented Software Architectures. In: The International Workshop on Predictor Models in Software Engineering (PROMISE 2007) (2007)

3. Qian, K., Liu, J., Tsui, F.: Decoupling Metrics for Services Composition. In: Proceedings of the 5th IEEE/ACIS International Conference and Information Sciences and 1st IEEE/ACIS International Workshop on Component-Based Software Engineering, Software Architecture and Reuse (ICIS-COMSAR 2006) (2006)

4. Arsanjani, A., Allam, A.: Service-Orineted Modeling and Architecture for Realization of an SOA. In: IEEE International Conference on Services Computing (SCC 2006) (2006)

5. Arsanjani, A., Zhang, L., Ellis, M., Allam, A., Channabasavaiah, K.: S3: A ServiceOriented Reference Architecture. IT Pro., 10-17 (June 2007)

6. Chidamber, S.R., Kemerer, C.F.: A Metrics Suite for Object Oriented Design. IEEE Transactions on Software Engineering 20(6), 476-493 (1994)

7. Arsanjani, A., Ghosh, S., Allam, A., Abdollah, T., Ganapathy, S., Holley, K.: SOMA: A method for developing service-oriented solutions. IBM Systems Journal 47(3), 377-396 (2008)

8. Web Services Resource Framework (WSRF), http://docs.oasis-open.org/wsrf/ wsrf-primer-1.2-primer-cd-02.pdf

9. Newcomer, E., Lomow, G.: Understanding SOA with Web Services. Addison Wesley, San Francisco (2005)

10. OASIS Web Services Transaction (WS-TX) landing page, http://www. oasis-open. org / committees / tc_home.php?wg_abbrev=ws-tx

11. Ferguson, D.F., Stockton, M.L.: Service Oriented Architecture: Programming Model and Product Architecture. IBM Systems Journal 44(4), 753-780 (2005)

12. Web Services Context Specification (WS-Context), http: //xml . coverpages .org/WS-ContextCD-9904.pdf 